\title{
Diaphragm ultrasound as a better predictor of successful extubation from mechanical ventilation than rapid shallow breathing index
}

\author{
Mohammad Jhahidul Alam', Simanta Roy ${ }^{2}$, Mohammad Azmain Iktidar², Fahmida Khatun Padma ${ }^{3}$, \\ Khairul Islam Nipun ${ }^{2}$, Sreshtha Chowdhury ${ }^{2}$, Ranjan Kumar Nath ${ }^{3}$, Harun-Or Rashid ${ }^{3}$ \\ ${ }^{1}$ Department of Critical Care Medicine, Imperial Hospital, Chattogram; ${ }^{2}$ Department of Public Health, North South University, Dhaka; ${ }^{3}$ Department of Anaesthesia \\ and Intensive Care Medicine, Chattogram Medical College Hospital, Chattogram, Bangladesh
}

Background: In 3\%-19\% of patients, reintubation is needed 48-72 hours following extubation, which increases intensive care unit (ICU) morbidity, mortality, and expenses. Extubation failure is frequently caused by diaphragm dysfunction. Ultrasonography can be used to determine the mobility and thickness of the diaphragm. This study looked at the role of diaphragm excursion (DE) and thickening fraction in predicting successful extubation from mechanical ventilation.

Methods: Thirty-one patients were extubated with the advice of an ICU consultant using the ICU weaning regimen and diaphragm ultrasonography was performed. Ultrasound DE and thickening fraction were measured three times: at the commencement of the T-piece experiment, at $10 \mathrm{~min}$ utes, and immediately before extubation. All patients' parameters were monitored for 48 hours after extubation. Rapid shallow breathing index (RSBI) was also measured at the same time.

Results: Successful extubation was significantly correlated with $\mathrm{DE}(\mathrm{P}<0.001)$. Receiver curve analysis for DE to predict successful extubation revealed good properties (area under the curve [AUC], 0.83; P<0.001); sensitivity, 77.8\%; specificity, 84.6\%; positive predictive value (PPV), 84.6\%; negative predictive value (NPV), 73.3\% while cut-off value, $11.43 \mathrm{~mm}$. Diaphragm thickening fraction (DTF) also revealed moderate curve properties (AUC, 0.69; $\mathrm{P}=0.06$ ); sensitivity, $61.1 \%$; specificity, 84.6\%; PPV, 87.5\%; NPV, 61.1\% with cut-off value 22.33\% although former one was slightly better. RSBI could not reach good receiver operating characteristic value at cut-off points 100 breaths/min/L (AUC, 0.58; $P=0.47$ ); sensitivity, 66.7\%; specificity, 53.8\%; PPV, 66.7\%; NPV, 53.8\%). Conclusions: To decrease the rate of reintubation, DE and DTF are better indicators of successful extubation. DE outperforms DTF.

Key Words: airway extubation; diaphragm excursion; diaphragm thickening fraction; rapid shallow breathing index; ventilator weaning

\section{INTRODUCTION}

Extubation failure is linked with a high mortality rate and adverse consequences such as aspiration, atelectasis, and pneumonia [1]. Therefore, before extubating a patient on artificial ventilation, the physician must determine whether they can breathe independently. This determination is made based on the results of a spontaneous breathing trial (SBT) using a

\section{Original Article}

Received: September 25, 2021

Revised: November 19, 2021

Accepted: November 20, 2021

Corresponding author

Mohammad Jhahidul Alam

Department of Critical Care

Medicine, Imperial Hospital, Zakir

Hossain Rd, Chattogram 4202,

Bangladesh

Tel: +880-18-1627-8447

Fax: +880-3161-0022

E-mail:alam2786@yahoo.com

Copyright (C) 2022 The Korean Society of Critical Care Medicine

This is an Open Access article distributed under the terms of Creative Attributions Non-Commercial License (https:// creativecommons.org/li-censes/by-nc/4.0/) which permits unrestricted noncommercial use, distribution, and reproduction in any medium, provided the original work is properly cited. 
T-piece or low-level pressure support. If the patient can spontaneously breathe, the physician must determine if the patient can undergo extubation [2]. Intensivists use four distinct methods when doing the SBT: (1) T-piece trial, in which only supplementary oxygen is delivered through a T-piece linked to an endotracheal tube; (2) continuous positive airway pressure (CPAP) trial, in which CPAP is set to the same level as the prior positive end-expiratory pressure (PEEP); (3) spontaneous trial with invasive ventilation with inspiratory pressure augmentation; and (4) automatic tube compensation [3].

Continuous or repetitive monitoring of vital parameters is required in these patients, and a combination of subjective and objective criteria is typically used to determine disease reversal. Oxygenation, hemodynamic status, acid-base balance, renal function, nutrition, and gastrointestinal function are monitored continuously or repetitively. However, it has been shown that assessing respiratory muscles, especially the diaphragm is inadequate in everyday intensive care unit (ICU) practice [4]. There is growing recognition that diaphragm weakening is prevalent in patients undergoing mechanical ventilation (MV) and is almost certainly a factor in extubation failure [5]. Recent researches indicate that the ventilator is likely to be the source of the reduced diaphragm force-generating capacity seen in mechanically ventilated patients, a condition called ventilator-induced diaphragmatic dysfunction $[6,7]$.

Weaning predictors are factors used to assist doctors in determining whether or not extubation efforts will be effective. Although an international consensus meeting in 2005 recommended against their regular use in clinical decision-making, researchers did not abandon the use [3]. Nevertheless, some individuals who do not meet all requirements are ultimately weaned [8]. It is estimated that $40 \%$ of the time of MV is spent on weaning to extubate the patients effectively [3]. Numerous weaning indices have been evaluated to determine the optimum weaning timeframe to avoid reintubation. Among these, the rapid shallow breathing index (RSBI) has gained widespread usage owing to its simplicity of computation and avoidance of complicated pulmonary mechanics calculations [9]. However, there are limitations in using RSBI to predict successful extubation as it does not consider the independent contribution of the diaphragm and is influenced by the accessory muscles of respiration [9]. There are also other predictors of successful extubation. These include respiratory rate alone, vital capacity, tidal volume per kilogram of body weight, airway occlusion pressure at 0.1 seconds, and an integrated evaluation of dynamic compliance, respiratory rate, oxygenation,

\section{KEY MESSAGES}

- Diaphragm ultrasonography (diaphragm excursion and diaphragm thickening fraction) is a better tool to predict successful extubation compared to rapid shallow breathing index.

- Among these two parameters, diaphragm excursion is a better predictor than diaphragm thickening fraction.

and maximum inspiratory pressure, referred to as the CROP (compliance, rate, oxygenation, and pressure) index, as well as other integrated weaning indices that utilize dynamic analogs. But all of them are influenced by different clinical states and have different cut-off values, sensitivities, and specificities. Respiratory rate can be increased by distress, pain, and acidosis and reduced by sedation and muscle paralysis, so it may not accurately reflect respiratory muscle strength and load. Vital capacity cannot be accurately measured in many clinical situations, such as reduced patient consciousness. Maximum inspiratory pressure has a significant disadvantage due to different measurement methods, which can give different values in other patients due to the use of analog or digital vacuum manometers [10].

The most often seen reason for extubation failure is diaphragmatic dysfunction, which increases over time while the patient is on MV [11]. So a single predictor which can be applicable in a wide range of clinical states and also gives an accurate picture of the activity of the diaphragm can help in predicting extubation failure. Ultrasound of the diaphragm is a non-invasive, straightforward procedure that is highly reproducible in the same individuals. As a result of its extensive usage, several criteria for diaphragmatic ultrasonography have been defined, including diaphragm excursion (DE) and diaphragm thickening fraction (DTF) [12]. Other diaphragmatic assessment techniques, such as fluoroscopy, phrenic nerve conduction, and trans-diaphragmatic pressure measurements, have limitations and drawbacks, particularly in the ICU, due to ionizing radiation exposure, lack of widely available methods in practice, and the requirement for patient transportation to the radiology department investigation room $[13,14]$.

Successful extubation to prevent reintubation is critically important to reduce mortality, morbidity, and expense of the patients. Among the conventional parameters, RSBI has gained the highest predictability, but its accuracy has been questioned. Since The diaphragm, the primary respiratory 
muscle, is not assessed during RSBI measurement, diaphragm dysfunction has often been missed; which later contributes to extubation failure with subsequent reintubation. Therefore, the aim of this study is to evaluate whether ultrasound measurements of DE and DTF are accurate predictors of successful extubation from the ventilator throughout the phases of weaning and whether they can make better prediction of effective extubation compared to RSBI.

\section{MATERIALS AND METHODS}

The study was approved by the Ethical Review Board (No. CMC/PG/2019/57) of the Chattogram Medical College Hospital, Chattogram, Bangladesh. Written informed consent was obtained from the patient's attendant.

\section{Study Participants}

From September 2019 to August 2020, this longitudinal observational research was conducted in the adult ICU of a tertiary level hospital in Bangladesh. During this period, 189 patients were assessed for eligibility and 33 of them met the inclusion criteria and their attendant provided informed written consent. But two patients died after enrollment, therefore, this study comprised a total of 31 intubated patients who were scheduled to be extubated according to their protocol.

Patients aged $>18$ years who had an adequate cough, no excessive tracheobronchial secretion, resolution of an underlying critical illness for which the patient was intubated \& was alert and cooperative without sedatives, hemodynamic stability (i.e., heart rate $140 \mathrm{bpm}$, mean arterial pressure $>65 \mathrm{~mm} \mathrm{Hg}$ without or with the lowest dose of vasopressors), stable metabolic status, and improved respiratory function: arterial oxygen saturation $\left(\mathrm{SaO}_{2}\right)>90 \%$ on fractional inspired oxygen $\left(\mathrm{FiO}_{2}\right) 0.4$ or partial pressure of oxygen $\left(\mathrm{PaO}_{2}\right) / \mathrm{FiO}_{2}>150 \mathrm{~mm} \mathrm{Hg}$, PEEP $8 \mathrm{~cm} \mathrm{H}_{2} \mathrm{O}$, respiratory rate $35 \mathrm{bpm}$ and without respiratory acidosis was included in this study. Patients having a history of diaphragm illness, cervical spine damage, neuromuscular disorders, a current thoracostomy, pneumothorax, pneumomediastinum, or any skin breach prohibiting ultrasound tests, or phrenic nerve palsy and patients' attendants refusing written consent was excluded from the research.

\section{Study Procedure}

The decision to discontinue SBT and reintubation of the patient was taken by the ICU consultant. We were informed when the ICU consultant took the extubation decision. Just at the start of 30 minutes of T-piece trial and after 10 minutes, right DE and DTF were evaluated using Sonosite M-Turbo (FUJIFILM SonoSite, Bothell, WA, USA) ultrasonography machine with the patient in semirecumbent position with the bed elevated between $20^{\circ}$ and $40^{\circ}$. Measurement of DE and DTF was recorded in a data sheet. RSBI was also simultaneously calculated at the bedside. The same procedure was done just before extubation. ICU consultant was unaware of ultrasonography results. Three measurements were taken during the T-piece trial and were averaged.

When the patient passed the 30 minutes of T-piece trial without deterioration, extubation was done by ICU consultant and received oxygen through nasal cannula patient was followed up for 48 hours post-extubation with regular checking of vital parameters and accordingly the post-extubation protocol to monitor the patient of the ICU of study institute. Extubation success is defined as the continuation of spontaneous breathing for at least 48 hours after extubation. Extubation failure was defined as the inability to breathe spontaneously for at least 48 hours without the assistance of a ventilator. For the patients who needed reintubation, their DE and DTF measurement measured during the T-piece trial was correlated with RSBI.

Diaphragm ultrasonic measurements were obtained in both brightness (B) and motion (M) mode on the right subcostal side. We utilized a high-resolution linear probe operating at 13-6 $\mathrm{MHz}$ and a curvilinear probe operating at $5-2 \mathrm{MHz}$ to determine the diaphragm thickness (DT) and DE in both B and $\mathrm{M}$ modes. According to the inclusion criteria, all patients were measured in the semirecumbent posture with the head of the bed raised between $20^{\circ}$ and $40^{\circ}$. A 5-2 $\mathrm{MHz}$ ultrasonic probe is used to determine the right $\mathrm{DE}$. The probe is positioned directly under the right costal edge along the mid- clavicular line and directed medially, cephalad, and dorsally such that the ultrasonic beam reaches the posterior part of the diaphragm perpendicularly. The liver serves as an acoustic window for the body. To begin, B-mode is utilized to get the best picture possible and to choose the exploration line.

Then, M-mode is used to see the diaphragm's motion along the chosen line. The normal diaphragm contracts and travels caudally toward the probe during inspiration; during expiration, the normal diaphragm contracts and moves cranially away from the probe; this is recorded as an upward motion of the M-mode tracing. The vertical axis of the tracing is used to quantify the DE from the baseline to the point of greatest inspiration height on the picture. Three measurements were taken, and the average was calculated. 
The DTF was determined using a 10-MHz linear ultrasound probe in the diaphragm and rib cage zone between the eighth and tenth intercostal spaces. The ultrasound picture is turned into a B-mode image. This region sees the diaphragm as a three-layer structure composed of two parallel echoic lines (the diaphragmatic pleura and the peritoneal membrane) and a hypoechoic structure in between (the muscle itself). At the end of expiration and inspiration, the picture was frozen. The DT on frozen images is the distance between the center of the pleural line and the middle of the peritoneal line. On the same scan, the DT was measured three times, and the results were averaged. The DTF was calculated as follows: DTF=(thickness at end inspiration-thickness at end-expiration)/thickness. The DTF was computed for each subject as the mean of the measured values. RSBI was collected concurrently with right DE and DTF measurements.

\section{Statistical Analysis}

Categorical data were presented as frequency \& relative frequency. The association between extubation outcome and RSBI, DE, DTF was analyzed using an independent sample t-test. Receiver operating characteristic (ROC) curves were used to assess the DE, DTF, and RSBI to predict successful extubation. Sensitivity and specificity were also analyzed to determine appropriate cut-off values of DE, DTF, and RSBI. Differences of the area under ROC curves were compared using the non-parametric method. A two-tailed P-value $<0.05$ is considered statistically significant. All statistical analyses were performed using Stata ver. 16 (StataCorp., College Station, TX, USA).

\section{RESULTS}

The baseline characteristics of the patients are presented in Table 1 . Of the 31 patients, the majority were above 50 years of age (35\%) and male (63\%). Hypertension (45\%), chronic obstructive pulmonary disease (32\%), and diabetes $(29 \%)$ were the predominant comorbidities, while $45 \%$ of the patients had no comorbidity. The patients were under invasive MV for an average of 11 days (standard deviation, 0.81 ) before the weaning trial. Eighteen patients (58\%) were successfully extubated, and $13(42 \%)$ needed reintubation. Of those who needed reintubation, most of them were required in 12 hours (19\%).

Table 2 presents the bivariate relationship between baseline and clinical parameters with extubation outcome. Patients with no comorbidity and a shorter duration of stay in MV were more likely to be extubated successfully. DE and DTF also showed a highly significant association, respectively, with successful extubation, whereas RSBI had no significant association.

The ROC curve for RSBI, DE and DTF is depicted in Figure 1. The area under the curve (AUC) for DE was high (0.83), and the P-value was highly significant $(<0.001)$. Both lower and upper bound area was also above the area of 0.5 indicating that DE could accurately predict the successful extubation. The AUC of DTF and RSBI was 0.69 (95\% confidence interval [CI], 0.49-0.88) and 0.58 (95\% CI, 0.36-0.80). Since the lower bound of the $95 \%$ confidence interval was below 0.5 for these two parameters with insignificant $\mathrm{P}$-value (DTF, $\mathrm{P}=0.06$ and RSBI, $\mathrm{P}=0.47$ ), they are poor predictors of successful extubation outcome. However, DTF is very close to a significant level. Table 3 represents the cut-off value, sensitivity, specificity, positive predictive value (PPV), and negative predictive value (NPV) for RSBI, DE, and DTF.

\section{DISCUSSION}

In this study, it is found that DE has a significant association

Table 1. Baseline characteristics of all patients

\begin{tabular}{lc}
\hline Characteristics & Value $(n=31)$ \\
\hline Age (yr) & $42 \pm 16$ \\
$<30$ & $7(23)$ \\
$30-39$ & $9(29)$ \\
$40-49$ & $4(13)$ \\
$\geq 50$ & $11(35)$ \\
Sex & \\
Male & $19(61)$ \\
Female & $12(39)$ \\
Comorbidity & \\
No comorbidity & $14(45)$ \\
Chronic kidney disease & $2(6)$ \\
Chronic obstructive pulmonary disease & $10(32)$ \\
Hypertension & $14(45)$ \\
Diabetes & $9(29)$ \\
Other & $1(3)$ \\
Duration of stay in mechanical ventilation (day) & $11.2 \pm 0.8$ \\
Extubation outcome & \\
Successful extubation & $18(58)$ \\
Reintubation needed in 6 hours & $4(13)$ \\
Reintubation needed in 12 hours & $6(19)$ \\
Reintubation needed in 24 hours & $3(10)$ \\
\hline
\end{tabular}

Values are presented as mean \pm standard deviation or number (\%). 
Table 2. Comparison of baseline and clinical parameters according to extubation outcome

\begin{tabular}{|c|c|c|c|}
\hline Variable & Successful extubation $(n=18)$ & Failed extubation $(n=13)$ & P-value \\
\hline Age (yr) & $38 \pm 14$ & $48 \pm 17$ & 0.07 \\
\hline Sex & & & 0.59 \\
\hline Male & $10(53)$ & $9(47)$ & \\
\hline Female & $8(67)$ & $4(33)$ & \\
\hline Comorbidity & & & 0.04 \\
\hline No comorbidity & $11(78.57)$ & $3(21.43)$ & \\
\hline One or more comorbidity & $7(41.18)$ & $10(58.82)$ & \\
\hline Duration of stay in mechanical ventilation (day) & $8.67 \pm 2.74$ & $14.62 \pm 4.33$ & $<0.001$ \\
\hline RSBI (breaths/min/L) & $100.46 \pm 2.84$ & $99.49 \pm 3.71$ & 0.41 \\
\hline $\mathrm{DE}(\mathrm{mm})$ & $12.41 \pm 2.38$ & $9.20 \pm 1.87$ & $<0.001$ \\
\hline DTF $(\%)$ & $22.34 \pm 2.73$ & $14.74 \pm 6.89$ & 0.04 \\
\hline
\end{tabular}

Values are presented as mean \pm standard deviation or number (\%).

RSBI: rapid shallow breathing index; DE: diaphragm excursion; DTF: diaphragm thickening fraction.

Table 3. Sensitivity, specificity, PPV, and NPV of RSBI, DE, and DTF to predict successful extubation

\begin{tabular}{lccccc}
\hline Parameter & Cut-off & Sensitivity $(\%)$ & Specificity $(\%)$ & PPV $(\%)$ & NPV $(\%)$ \\
\hline RSBI (breaths/min/L) & 100 & 66.7 & 53.8 & 66.7 & 53.8 \\
DE $(\mathrm{mm})$ & 11.43 & 77.8 & 84.6 & 87.5 & 73.3 \\
DTF $(\%)$ & 22.33 & 61.1 & 84.6 & 84.6 & 61.1 \\
\hline
\end{tabular}

PPV: positive predictive value; NPV: negative predictive value; RSBI: rapid shallow breathing index; DE: diaphragm excursion; DTF: diaphragm thickening fraction.

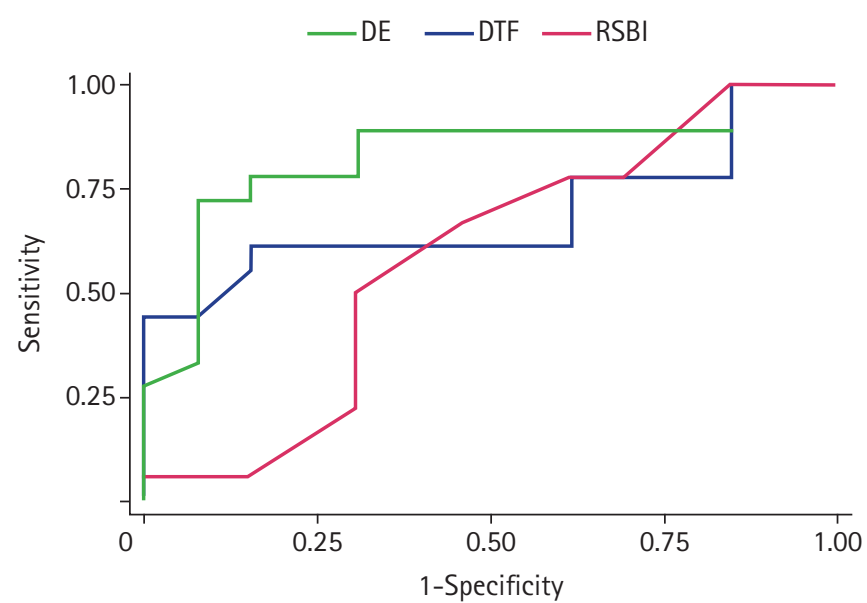

Figure 1. Comparison of receiver operating characteristic (ROC) curve for diaphragm excursion (DE), diaphragm thickening fraction (DTF), and rapid shallow breathing index (RSBI). Area under the ROC curves for DE: 0.83 (95\% confidence interval [Cl], 0.68-0.99), for DTF: 0.69 (95\% Cl, 0.49-0.88), and for RSBI: 0.58 (95\% Cl, 0.36-0.80).

with extubation success. DE predicted successful extubation in this study which matched with several studies [15-17]. DE showed excellent properties in the ROC curve. The AUC was 0.83 with significant value, while cut-off value $11.43 \mathrm{~mm}$, sensitivity revealed $77.8 \%$, specificity $84.6 \%$, PPV $87.5 \%$ and NPV
73.3\%. Similar result was found in the study of Spadaro et al. [18] with cut-off value of $\leq 14 \mathrm{~mm}$, AUC, 0.82 , sensitivity, $88.2 \%$, specificity, 61.8\%, PPV, 53.6\% and NPV, 91.3\% [18]. Farghaly and Hasan [19] evaluated diaphragmatic parameters (DT, DTF and DE) in 54 patients who successfully passed SBT. He found a sensitivity of $87.5 \%$ and specificity of $71.2 \%$ when the cut-off value of DE was $\geq 10.5 \mathrm{~mm}$ with AUC 0.879 .

Osman and Hashim [16] found a cut-off value of $10 \mathrm{~mm}$; sensitivity, 83.3\%; specificity, 100\%; NPV, 94.3\%; and PPV, $100 \%$. DTF was not significantly correlated with successful extubation in this study but very close to a significant level. However, it was found significant in some. The DTF scores showed good properties with AUC 0.706; cut-off value, 19.77; sensitivity, $58.8 \%$; specificity, $77.8 \%$; PPV, $83.3 \%$; and NPV, $50.0 \%$ in this study. A better result was observed about DTF in the study of Osman and Hashim [16], with a $28 \%$ cut-off value showed $88.9 \%$ sensitivity, $100 \%$ specificity, $96.2 \%$ NPV, and $100 \%$ PPV. Osman and Hashim [16] investigated and wrote a review article on the efficacy of DTF where he explained that cut-off for DTF varied $30-34$ and sensitivity varied $88 \%-90 \%$, and specificity ranged $61 \%-82 \%$. The insignificant correlation of DTF with successful extubation in this study is due to the low sample size. Zambon et al. [20] stated a lack of data 
about the learning curve to measure the thickening fraction to operator-dependent variations, which influence the measurements.

RSBI was shown to be a poor predictor of extubation success in our study. Karthika et al. [9] showed that the RSBI should not be used generally to predict effective extubation. Danaga et al. [21] discovered that the conventional RSBI cut-off value (105 breaths/min/L) accurately predicted just $20 \%$ of extubation failures. According to Boutou et al. [22], RSBI measurements taken early in an SBT cannot correctly predict successful extubation. RSBI is not a reliable predictor of effective extubation in neurosurgical patients, as discovered by Vidotto et al. [23]. According to Verceles et al. [24], isolated RSBI measures do not reliably predict successful extubation in patients requiring prolonged MV. According to Teixeira et al. [25], serial RSBI measurements throughout 120 minutes of SBT were unable to identify extubation failure in patients who had previously had a successful SBT with an initial RSBI of 105 breaths/min/L. According to Shah et al. [26], RSBI does not vary substantially throughout a 90-minute SBT during the trial.

In a study, Spadaro et al. [18] found that DE is better than RSBI. Another study by Dinino et al. [27] found that the DTF is better than RSBI. This study found that both DE and DTF were better at predicting successful extubation than RSBI, as the former had better ROC properties. Among these two parameters, DE is better than DTF.

Ultrasonography can help diagnose diaphragm dysfunction and determine whether or not extubation will be successful. DE and DTF can therefore be useful parameters in predicting extubation success. Among these two, DE outperformed DTF in predicting successful extubation outcomes.

Since this study is single-centric with a small sample size, the results may not be generalizable, and selection bias cannot be ruled out completely. Furthermore, we did not compare diaphragm strength to sonographic findings by magnetic phrenic nerve stimulation, which is considered the gold standard [28].

Ultrasound-based diaphragm measurements, mainly DE and DTF, are significant predictors of successful extubation than traditional parameters like RSBI. So it should be routinely done in the ICU. Future studies in multiple centers with larger sample sizes should be conducted to verify the results.

\section{CONFLICT OF INTEREST}

No potential conflict of interest relevant to this article was reported.

\section{ORCID}

Mohammad Jhahidul Alam

https://orcid.org/0000-0003-3698-2467

Simanta Roy https://orcid.org/0000-0002-7124-5043

Mohammad Azmain Iktidar

https://orcid.org/0000-0002-9073-5451

Fahmida Khatun Padma https://orcid.org/0000-0001-9109-1208

Khairul Islam Nipun https://orcid.org/0000-0002-4740-0349

Sreshtha Chowdhury https://orcid.org/0000-0003-3482-8204

Ranjan Kumar Nath ～https://orcid.org/0000-0003-1141-2994

Harun-Or Rashid https://orcid.org/0000-0002-0106-8168

\section{AUTHOR CONTRIBUTIONS}

Conceptualization: MJA. Data curation: FKP. Formal analysis: MAI, SR. Methodology: MJA, SC. Project administration: MJA, KIN. Visualization: MJA, KIN. Writing-original draft: MJA, SR. Writing-review \& editing: RKN, HOR.

\section{REFERENCES}

1. Kulkarni AP, Agarwal V. Extubation failure in intensive care unit: predictors and management. Indian J Crit Care Med 2008;12:1-9

2. Mokhlesi B, Tulaimat A, Gluckman TJ, Wang Y, Evans AT, Corbridge TC. Predicting extubation failure after successful completion of a spontaneous breathing trial. Respir Care 2007;52:1710-7

3. Zein H, Baratloo A, Negida A, Safari S. Ventilator weaning and spontaneous breathing trials; an educational review. Emerg (Tehran) 2016;4:65-71.

4. Sigala I, Vassilakopoulos T. Diaphragmatic ultrasound as a monitoring tool in the intensive care unit. Ann Transl Med 2017; 5:79.

5. Jung B, Moury PH, Mahul M, de Jong A, Galia F, Prades A, et al. Diaphragmatic dysfunction in patients with ICU-acquired weakness and its impact on extubation failure. Intensive Care Med 2016;42:853-61.

6. Liu YY, Li LF. Ventilator-induced diaphragm dysfunction in critical illness. Exp Biol Med (Maywood) 2018;243:1329-37.

7. Petrof BJ, Hussain SN. Ventilator-induced diaphragmatic dysfunction: what have we learned? Curr Opin Crit Care 2016;22:67-72.

8. Ely EW, Baker AM, Evans GW, Haponik EF. The prognostic significance of passing a daily screen of weaning parameters. 
Intensive Care Med 1999;25:581-7.

9. Karthika M, Al Enezi FA, Pillai LV, Arabi YM. Rapid shallow breathing index. Ann Thorac Med 2016;11:167-76.

10. Nemer SN, Barbas CS. Predictive parameters for weaning from mechanical ventilation. J Bras Pneumol 2011;37:669-79.

11. Goligher EC, Fan E, Herridge MS, Murray A, Vorona S, Brace D, et al. Evolution of diaphragm thickness during mechanical ventilation. impact of inspiratory effort. Am J Respir Crit Care Med 2015;192:1080-8.

12. Mayo P, Volpicelli G, Lerolle N, Schreiber A, Doelken P, Vieillard-Baron A. Ultrasonography evaluation during the weaning process: the heart, the diaphragm, the pleura and the lung. Intensive Care Med 2016;42:1107-17.

13. Matamis D, Soilemezi E, Tsagourias M, Akoumianaki E, Dimassi S, Boroli F, et al. Sonographic evaluation of the diaphragm in critically ill patients: technique and clinical applications. Intensive Care Med 2013;39:801-10.

14. Pirompanich P, Romsaiyut S. Use of diaphragm thickening fraction combined with rapid shallow breathing index for predicting success of weaning from mechanical ventilator in medical patients. J Intensive Care 2018;6:6.

15. Ali ER, Mohamad AM. Diaphragm ultrasound as a new functional and morphological index of outcome, prognosis and discontinuation from mechanical ventilation in critically ill patients and evaluating the possible protective indices against VIDD. Egypt J Chest Dis Tuberc 2017;66:339-51.

16. Osman AM, Hashim RM. Diaphragmatic and lung ultrasound application as new predictive indices for the weaning process in ICU patients. Egypt J Radiol Nucl Med 2017;48:61-6.

17. Yoo JW, Lee SJ, Lee JD, Kim HC. Comparison of clinical utility between diaphragm excursion and thickening change using ultrasonography to predict extubation success. Korean J Intern Med 2018;33:331-9.

18. Spadaro S, Grasso S, Mauri T, Dalla Corte F, Alvisi V, Ragazzi R, et al. Can diaphragmatic ultrasonography performed during the T-tube trial predict weaning failure? The role of diaphragmatic rapid shallow breathing index. Crit Care 2016;20:305.

19. Farghaly S, Hasan AA. Diaphragm ultrasound as a new method to predict extubation outcome in mechanically ventilated patients. Aust Crit Care 2017;30:37-43.

20. Zambon M, Greco M, Bocchino S, Cabrini L, Beccaria PF, Zangrillo A. Assessment of diaphragmatic dysfunction in the critically ill patient with ultrasound: a systematic review. Intensive Care Med 2017;43:29-38.

21. Danaga AR, Gut AL, Antunes LC, Ferreira AL, Yamaguti FA, Christovan JC, et al. Evaluation of the diagnostic performance and cut-off value for the rapid shallow breathing index in predicting extubation failure. J Bras Pneumol 2009;35:541-7.

22. Boutou AK, Abatzidou F, Tryfon S, Nakou C, Pitsiou G, Argyropoulou P, et al. Diagnostic accuracy of the rapid shallow breathing index to predict a successful spontaneous breathing trial outcome in mechanically ventilated patients with chronic obstructive pulmonary disease. Heart Lung 2011;40:105-10.

23. Vidotto MC, Sogame LC, Calciolari CC, Nascimento OA, Jardim JR. The prediction of extubation success of postoperative neurosurgical patients using frequency-tidal volume ratios. Neurocrit Care 2008;9:83-9.

24. Verceles AC, Diaz-Abad M, Geiger-Brown J, Scharf SM. Testing the prognostic value of the rapid shallow breathing index in predicting successful weaning in patients requiring prolonged mechanical ventilation. Heart Lung 2012;41:546-52.

25. Teixeira C, Zimermann Teixeira PJ, Hohër JA, de Leon PP, Brodt SF, da Siva Moreira J. Serial measurements of f/VT can predict extubation failure in patients with $\mathrm{f} / \mathrm{VT}<$ or $=105$ ? J Crit Care 2008;23:572-6.

26. Shah NG, Lee B, Colice G. Analysis of rapid shallow breathing index as a predictor for successful extubation from mechanical ventilation. Chest 2004;126:756S

27. DiNino E, Gartman EJ, Sethi JM, McCool FD. Diaphragm ultrasound as a predictor of successful extubation from mechanical ventilation. Thorax 2014;69:423-7.

28. Demoule A, Jung B, Prodanovic H, Molinari N, Chanques G, Coirault C, et al. Diaphragm dysfunction on admission to the intensive care unit: prevalence, risk factors, and prognostic impact-a prospective study. Am J Respir Crit Care Med 2013;188:213-9. 